Rudolf Rainer(- · Jarkko Siltakoski · Thomas Stanin (1)

\title{
An evolutionary Haar-Rado type theorem
}

Received: 18 August 2020 / Accepted: 13 March 2021 / Published online: 3 April 2021

\begin{abstract}
In this paper, we study variational solutions to parabolic equations of the type $\partial_{t} u-\operatorname{div}_{x}\left(D_{\xi} f(D u)\right)+D_{u} g(x, u)=0$, where $u$ attains time-independent boundary values $u_{0}$ on the parabolic boundary and $f, g$ fulfill convexity assumptions. We establish a HaarRado type theorem: If the boundary values $u_{0}$ admit a modulus of continuity $\omega$ and the estimate $\left|u(x, t)-u_{0}(\gamma)\right| \leq \omega(|x-\gamma|)$ holds, then $u$ admits the same modulus of continuity in the spatial variable.
\end{abstract}

\section{Introduction}

In this article, we are concerned with establishing a Haar-Rado type theorem for the following kind of Cauchy-Dirichlet problem:

$$
\left\{\begin{array}{cc}
\partial_{t} u-\operatorname{div}_{x}\left(D_{\xi} f(D u)\right)+D_{u} g(x, u)=0 & \text { in } \Omega_{T}, \\
u=u_{0} & \text { on } \partial_{P} \Omega_{T},
\end{array}\right.
$$

for a time-independent boundary datum $u_{0}: \bar{\Omega} \rightarrow \mathbb{R}$. For $T>0$, the set $\Omega_{T}:=\Omega \times$ $(0, T)$ is a space-time cylinder with a bounded domain $\Omega \subset \mathbb{R}^{n}, n \geq 2$. As usual, by $\partial_{P} \Omega_{T}:=(\bar{\Omega} \times\{0\}) \cup(\partial \Omega \times(0, T))$ we denote the parabolic boundary of $\Omega_{T}$, use the operator symbol $\partial_{t}$ to express a derivative with respect to the time variable, and write $\operatorname{div}_{x}$ for the spatial divergence operator.

The classical Haar-Rado theorem (see e.g. [15, Prop. 2.11] and the references therein) was originally formulated in an elliptic setting, which is concerned with the minimization problem

$$
\min \left\{\int_{\Omega} f(D u) \mathrm{d} x: u \in \operatorname{Lip}_{\phi}(\Omega)\right\},
$$

Rudolf Rainer ( $\varangle)$ : Fachbereich Mathematik, Universität Salzburg, Hellbrunner Str. 34, 5020 Salzburg, Austria

e-mail: rudolf.rainer@sbg.ac.at

Jarkko Siltakoski: Department of Mathematics and Statistics, University of Jyväskylä, P.O.Box 35, FIN-40014 Jyväskylä, Finland

e-mail: jarkko.siltakoski@jyu.fi

Thomas Stanin: Fachbereich Mathematik, Universität Salzburg, Hellbrunner Str. 34, 5020

Salzburg, Austria

e-mail: thomas.stanin@sbg.ac.at

Mathematics Subject Classification: 35A15 · 35B65 · 35K55 - 49J40 
where $f$ is strictly convex and $\operatorname{Lip}_{\phi}(\Omega)$ denotes the space of Lipschitz functions having boundary values equal to the given Lipschitz function $\phi$ on the boundary $\partial \Omega$ of an open and bounded set $\Omega \subset \mathbb{R}^{n}$. The theorem then states that a minimizer $u$ fulfills the property

$$
\sup _{x, y \in \Omega} \frac{|u(x)-u(y)|}{|x-y|}=\sup _{x \in \Omega, \gamma \in \partial \Omega} \frac{|u(x)-\phi(\gamma)|}{|x-\gamma|} .
$$

As seen in [15, Prop. 11.41], this can be used to obtain global gradient estimates on $\bar{\Omega}$ from mere boundary estimates on $\partial \Omega$ and it also plays a fundamental role in the existence proof (cf. [16, Lemma 1.3]) for Lipschitz minimizers to (1.2) whenever the boundary datum $\phi$ fulfills a barrier condition, such as the quite restrictive bounded slope condition (see Definition 2.7 and [19,27] for some discussion). This condition has a long tradition within a sub-field of Calculus of Variations, which is now known as Hilbert-Haar theory and is based on papers from Haar [17], Hartman and Nirenberg [20], Stampacchia [28], Miranda [27], and Hartman and Stampacchia [21]. In recent years, many authors were investigating questions considering existence and regularity of minimizers to a more general class of problems of the form

$$
\min \left\{\int_{\Omega} f(D u)+g(x, u) \mathrm{d} x: u \in W_{\phi}^{1, p}(\Omega)\right\},
$$

where - for a suitable domain $\Omega \subset \mathbb{R}^{n}$ and $p \geq 1-W_{\phi}^{1, p}(\Omega)$ denotes the space of Sobolev functions $u \in W^{1, p}(\Omega)$ having trace equal to $\phi$ on $\partial \Omega$. For a selection of results covering the case $g=0$, we refer the interested reader to $[6,10-12,24,25]$, as well as to $[7-9,14,23]$ for some results about the general case $g \neq 0$. In this context, the bounded slope condition was also considerably weakened in a few ways, one of them being a one-sided bounded slope condition (see [12]).

Following this approach, in [26] Mariconda and Treu reformulated and generalized the classical Haar-Rado theorem (1.3) to the new setting (1.4) for $p=1$, even allowing $\phi$ to admit a general modulus of continuity $\omega$.

More precisely, they showed that if, among other assumptions,

$$
|u(x)-\phi(\gamma)| \leq \omega(|x-\gamma|) \quad \text { for a.e. } x \in \Omega \text { and for all } \gamma \in \partial \Omega,
$$

this estimate can be extended to the interior of $\Omega$, i.e. $u$ has an $\omega$-continuous representative. Note that this is a reformulation of (1.3) in the case that $\omega$ is a Lipschitz-modulus.

Our main goal in this paper is to extend this statement to the parabolic setting, in terms of variational solutions. For a given variational solution $u$ to (1.1), attaining $\omega$-continuous and time-independent boundary data $u_{0}$ on the parabolic boundary, we show that if

$$
\left|u(x, t)-u_{0}\left(x_{0}\right)\right| \leq \omega\left(\left|x-x_{0}\right|\right) \quad \text { for a.e. }(x, t) \in \Omega_{T} \text { and for all } x_{0} \in \partial \Omega,
$$

then, as before, this estimate can be extended slice-wise to the interior of the spacetime cylinder $\Omega_{T}$, provided that the intersection of $\Omega$ with a shifted version of itself has a Lipschitz boundary. 
We point out again that we treat time-independent boundary data. From our point of view the investigation of boundary data which depend on time, i.e. $u_{0}=u_{0}(x, t)$ requires further investigation and could be an interesting subject for future studies.

Following the tradition of Hilbert-Haar theory, we will not suppose any growth conditions from above on $f$ or $g$. For this reason we cannot use the notion of weak solutions and instead take the so called variational approach in the spirit of Lichnewsky and Temam [22]. This approach to evolutionary equations has become increasingly popular in the last several years and the existence of variational solutions has been obtained to rather general equations, see for example [1-3].

Regularity of variational solutions was studied by Bögelein, Duzaar, Marcellini and Signoriello [4], who obtained the spatial Lipschitz continuity of solutions to (1.1) when $g \equiv 0$ and $u_{0}$ satisfies the bounded slope condition. Older results regarding regularity proofs of weak solutions via the bounded slope condition in an evolutionary setting with functionals of linear growth can for example be found in Hardt and Zhou [18, Chapter 4]. Moreover, recently Bögelein and Stanin [5] proved local Lipschitz continuity in spacetime of variational solutions under an evolutionary variant of the previously mentioned one-sided bounded slope condition.

Our article will be structured as follows: In Sect. 2 we present the setting and our main result. Several lemmata, which are useful for our purposes, are collected in Sect. 3. Then we proceed with the proof of our main result, which can be summarized as follows: We compare the variational solution $u$ with the shifted variational solution, defined on a shifted space-time cylinder. Topic of Sect. 4 is the proof that this shift retains the property of being a variational super- or sub-solution. In the main step, which is carried out in Sect. 5, we can thus apply a comparison principle on the intersection of the original cylinders. Finally, in Sect. 6 we apply our main result to obtain the spatial Lipschitz continuity of variational solutions in the setting of the bounded slope condition.

\section{Definitions and main result}

Throughout this article, we denote by $\Omega_{T}:=\Omega \times(0, T)$ a spacetime cylinder with $T>0$ and a bounded Lipschitz domain $\Omega \subset \mathbb{R}^{n}$. For a function $v \in L^{1}\left(\Omega_{T}\right) \equiv$ $L^{1}\left([0, T] ; L^{1}(\Omega)\right)$, we will often adopt the abbreviation $v(t):=v(\cdot, t)$ to denote the evaluation of the function $v$ at time $t \in[0, T]$, which is to be understood in a suitable sense.

We consider the Cauchy-Dirichlet problem (1.1) with two variational integrands $f, g$, which we impose different conditions on. For the (only gradient-dependent) variational integrand $f: \mathbb{R}^{n} \rightarrow \mathbb{R}$ we require that

$$
\left\{\begin{array}{c}
f \text { is convex, } \\
f \text { is } p \text {-coercive, i.e. } f(\xi) \geq \mu|\xi|^{p}+v \text { for all } \xi \in \mathbb{R}^{n} \text {, some } \mu>0 \text { and } v \in \mathbb{R},
\end{array}\right\}
$$


with an exponent $p>1$. For the ( $x$ - and $u$-dependent) variational integrand $g: \Omega \times \mathbb{R} \rightarrow \mathbb{R}$ we require that

$$
\left\{\begin{array}{c}
x \mapsto g(x, u) \text { is measurable for all } u \in \mathbb{R}, \\
u \mapsto g(x, u) \text { is convex for almost every } x \in \Omega, \\
g(x, u) \geq-k(x)(1+|u|) \text { for a.e. } x \in \Omega \text { and every } u \in \mathbb{R},
\end{array}\right\}
$$

where $k \in L^{p^{\prime}}\left(\Omega ; \mathbb{R}_{\geq 0}\right)$ with $p^{\prime}:=\frac{p}{p-1}$ denoting the conjugate Hölder exponent of $p$. Due to convexity, the right derivative of $g$ with respect to the second variable exists, is monotonically non-decreasing and we denote it by $g_{u}^{+}$. Furthermore, we impose the following conditions on the boundary datum $u_{0}$ :

$$
\left\{\begin{array}{c}
u_{0} \in W^{1, p}(\Omega) \cap L^{2}(\Omega), \\
\int_{\Omega} f\left(D u_{0}\right)+g\left(x, u_{0}\right) \mathrm{d} x<\infty
\end{array}\right\}
$$

As mentioned, we do not demand any growth conditions from above on neither $f$ nor $g$, and therefore we cannot use the notion of weak solutions in this context. Instead, we use variational solutions - a notion going back to the article [22] of Lichnewsky and Temam - whose existence to (1.1) is guaranteed due to our structural assumptions on $f, g$ and $u_{0}$ (cf. Theorem 2.2). If $f$ and $g$ are suitably regular, then variational solutions coincide with weak solutions [2].

In order to define variational solutions, we first restate the definition of typical function spaces in the parabolic setting: The parabolic Sobolev space $L^{p}\left([0, T] ; W^{1, p}(\Omega)\right)$ is the set of all measurable functions $u: \Omega_{T} \rightarrow \mathbb{R}$ such that $x \mapsto u(x, t)$ is in $W^{1, p}(\Omega)$ for a.e. $t \in(0, T)$ and further

$$
\int_{\Omega_{T}}|u|^{p}+|D u|^{p} \mathrm{~d} z<\infty .
$$

Similarly, for $v \in L^{p}\left([0, T] ; W^{1, p}(\Omega)\right)$ we denote by $v+L^{p}\left([0, T] ; W_{0}^{1, p}(\Omega)\right)$ the set of all measurable functions such that $x \mapsto u(x, t)-v(x, t)$ belongs to $W_{0}^{1, p}(\Omega)$ for almost every $t \in(0, T)$ and (2.4) is fulfilled.

Since we will also need weaker versions of the mentioned variational solutions later on, we define them in the following lines as well:

Definition 2.1. (Variational (sub-/super-) solution) We call a function

$$
u \in L^{p}\left([0, T] ; W^{1, p}(\Omega)\right) \cap L^{2}\left(\Omega_{T}\right) \text { with } \partial_{t} u \in L^{2}\left(\Omega_{T}\right)
$$

a variational sub-solution (respectively a variational super-solution) on $\Omega_{T}$ if

$$
\int_{\Omega_{T}} f(D u)+g(x, u) \mathrm{d} z<\infty
$$

and the inequality

$$
\begin{aligned}
\int_{\Omega_{T}} f(D u)+g(x, u) \mathrm{d} z \leq & \int_{\Omega_{T}} \partial_{t} v(v-u)+f(D v)+g(x, v) \mathrm{d} z \\
& -\left.\frac{1}{2} \int_{\Omega}(v-u)^{2}(x, \cdot) \mathrm{d} x\right|_{0} ^{T}
\end{aligned}
$$


holds true for any $v \in u+L^{p}\left([0, T] ; W_{0}^{1, p}(\Omega)\right) \cap L^{2}\left(\Omega_{T}\right)$ with $v \leq u$ almost everywhere in $\Omega_{T}$ (resp. $v \geq u$ almost everywhere in $\Omega_{T}$ ), additionally satisfying $\partial_{t} v \in L^{2}\left(\Omega_{T}\right)$. Furthermore, we call $u$ a variational solution if it is both variational sub- and super-solution. In this case, any $v \in u+L^{p}\left([0, T] ; W_{0}^{1, p}(\Omega)\right) \cap L^{2}\left(\Omega_{T}\right)$ with $\partial_{t} v \in L^{2}\left(\Omega_{T}\right)$ is an admissible test function. Finally, such $u$ is called a variational solution to the Cauchy-Dirichlet problem (1.1) if $u(x, t)=u_{0}(x)$ on the lateral boundary $\partial \Omega \times(0, T)$ in the sense of traces and $u(\cdot, 0)=u_{0}(\cdot)$.

Note that for a variational solution one usually demands that

$$
u \in L^{p}\left([0, T] ; W^{1, p}(\Omega)\right) \cap C^{0}\left([0, T] ; L^{2}(\Omega)\right),
$$

where $C^{0}\left([0, T] ; L^{2}(\Omega)\right)$ denotes the Bochner space of continuous functions $u$ : $[0, T] \rightarrow L^{2}(\Omega)$ such that

$$
\max _{t \in[0, T]} \int_{\Omega}|u(x, t)|^{2} \mathrm{~d} x<\infty .
$$

However, it is worth citing the existence and regularity result obtained in [2, Theorem 1.2]. It ensures existence of variational (sub- and super-)solutions and also includes important regularity properties. For the sake of completeness, we include the statement, adapted to our setting, which is a special case of the original Theorem.

Theorem 2.2. Let $f$ satisfy the conditions given in (2.1), $g$ the assumptions in (2.2) and suppose $u_{0}$ to fulfill the constraints in (2.3). Then, there exists a variational solution (and thus also a sub-resp. super-solution) $u \in L^{p}\left([0, T] ; W^{1, p}(\Omega)\right) \cap$ $C^{0}\left([0, T] ; L^{2}(\Omega)\right)$ to $(1.1)$, that further fulfils $u \in C^{0,1 / 2}\left([0, T] ; L^{2}(\Omega)\right)$ and admits a weak time derivative $\partial_{t} u \in L^{2}\left(\Omega_{T}\right)$.

The property $\partial_{t} u \in L^{2}\left(\Omega_{T}\right)$ will be an important technical tool in the proofs. Thus, we impose this condition in the definition above. Further note that the property $u \in C^{0}\left([0, T] ; L^{2}(\Omega)\right)$ follows from $\partial_{t} u \in L^{2}\left(\Omega_{T}\right)$ by using a standard mollifier argument.

Definition 2.3. (Modulus of continuity) A continuous and increasing function $\omega$ : $[0, \infty) \rightarrow[0, \infty)$ with $\omega(0)=0$ is called a modulus of continuity. For a set $X \subset$ $\mathbb{R}^{n}$, a function $\phi: X \rightarrow \mathbb{R}$ is called $\omega$-continuous if $|\phi(x)-\phi(y)| \leq \omega(|x-y|)$ for all $x, y \in X$.

Later, we will need the fact that shifting leaves the property of being a variational super-solution invariant (cf. Theorem 4.2). Due to the $x$-dependency of the function $g$, mere convexity is not enough to obtain this. We need the following stronger assumption: For $h \in \mathbb{R}^{n}, x \in \mathbb{R}$ and a modulus of continuity $\omega$, we demand

$$
v \geq u+\omega(|h|) \Rightarrow g_{u}^{+}(x-h, v) \geq g_{u}^{+}(x, u) \quad \forall x \in \mathbb{R}^{n}, \forall u, v \in \mathbb{R} . \quad\left(\mathrm{H}_{h, \omega}\right)
$$

If $g$ does not depend on $x$ and is convex, then this property is fulfilled. For another sufficient condition, see [26, Prop. 4.1]. 
We define the shifted set $\Omega_{h}:=\{x+h: x \in \Omega\}$ and denote the corresponding shifted spacetime cylinder by $\Omega_{h, T}:=\Omega_{h} \times(0, T)$. Additionally, for a function $u: \Omega_{T} \rightarrow \mathbb{R}$, we define the associated shifted function $u_{h}: \Omega_{h, T} \rightarrow \mathbb{R}$ via

$$
u_{h}(x, t):=u(x-h, t) .
$$

Now we are able to formulate our main result, which is the following:

Theorem 2.4. (Haar-Rado type) Suppose that the conditions (2.1) - (2.3) and $\left(\mathrm{H}_{h, \omega}\right)$ hold. Further suppose u to be a variational solution to (1.1) and assume $u_{0}$ to be $\omega$-continuous on $\bar{\Omega}$ with a given modulus of continuity $\omega$.

Additionally, let $\partial\left(\Omega \cap \Omega_{h}\right)$ be Lipschitz for all $h \in \mathbb{R}^{n}$ and suppose that the estimate

$$
\left|u(x, t)-u_{0}\left(x_{0}\right)\right| \leq \omega\left(\left|x-x_{0}\right|\right)
$$

holds true for any $x_{0} \in \partial \Omega$ and almost every $(x, t) \in \Omega_{T}$. Then, $u$ satisfies

$$
|u(y, t)-u(x, t)| \leq \omega(|y-x|),
$$

whenever $(x, t)$ and $(y, t) \in \Omega_{T}$ are Lebesgue points of $u$.

Note that the requirement that $\Omega$ has a Lipschitz boundary does not imply that $\partial\left(\Omega \cap \Omega_{h}\right)$ is Lipschitz. The reason for imposing the conditions on the boundary is to ensure the existence of the trace operator. For further details, see [26, Section 3.2].

Corollary 2.5. Suppose that the conditions (2.1) - (2.3) and $\left(\mathrm{H}_{h, \omega}\right)$ hold. Let $\partial(\Omega \cap$ $\Omega_{h}$ ) be Lipschitz. Suppose that $u$ is a variational solution to (1.1) on $\Omega_{T}$ with initial and boundary data $u_{0}$. Suppose that $u_{0}$ is $\omega$-continuous in $\bar{\Omega}$. Suppose that there exist $l_{1}, l_{2} \in W_{u_{0}}^{1, p}(\Omega)$ that are $\omega$-continuous on $\bar{\Omega}$ and

$$
l_{1} \leq u \leq l_{2} \text { in } \Omega_{T} .
$$

Then we have

$$
|u(y, t)-u(x, t)| \leq \omega(|y-x|)
$$

whenever $(x, t),(y, t) \in \Omega_{T}$ are Lebesgue points of $u$.

A straight-forward situation in which $\partial\left(\Omega \cap \Omega_{h}\right)$ is Lipschitz for any $h>0$, is if $\Omega$ is bounded and convex. We thus formulate the following corollary, with the often used Hölder-continuity:

Corollary 2.6. Suppose that the conditions (2.1) - (2.3) and $\left(\mathrm{H}_{h, \omega}\right)$ hold. Let $\Omega$ be a bounded and convex domain. Suppose that $u$ is a variational solution to (1.1) in $\Omega_{T}$ with initial and boundary data $u_{0}$. For $\alpha \in(0,1)$ let $u_{0}$ be $\alpha$-Hölder-continuous on $\bar{\Omega}$. Assume that

$$
\left|u(x, t)-u_{0}\left(x_{0}\right)\right| \leq\left|x-x_{0}\right|^{\alpha}
$$

holds true for any $x_{0} \in \partial \Omega$ and almost every $(x, t) \in \Omega_{T}$. Then, $u$ satisfies

$$
|u(y, t)-u(x, t)| \leq|y-x|^{\alpha},
$$

whenever $(x, t)$ and $(y, t) \in \Omega_{T}$ are Lebesgue points of $u$. 
As an application of our main result, we obtain the spatial Lipschitz continuity of variational solutions to (1.1) under certain assumptions. In particular, we suppose the so called bounded slope condition on the initial and boundary data $u_{0}$.

Definition 2.7. (Bounded slope condition) A function $u_{0}: \partial \Omega \rightarrow \mathbb{R}$ satisfies the bounded slope condition with constant $Q>0$ if for any $x_{0} \in \partial \Omega$ there exist affine functions $a_{1}, a_{2}: \mathbb{R}^{n} \rightarrow \mathbb{R}$ such that $\left|D a_{1}\right|,\left|D a_{2}\right|<Q, a_{1}\left(x_{0}\right)=u_{0}\left(x_{0}\right)=a_{2}\left(x_{0}\right)$ and $a_{1} \leq u_{0} \leq a_{2}$ on $\partial \Omega$.

The bounded slope condition forces $u_{0}$ to be an affine function on flat parts of $\partial \Omega$ and $\Omega$ to be convex unless $u_{0}$ is an affine function. If $\Omega$ is a uniformly convex and bounded $C^{2}$ domain, then $\left.u_{0}\right|_{\partial \Omega}$ satisfies the bounded slope condition whenever $u_{0} \in C^{2}\left(\mathbb{R}^{n}\right)$, see [16,27] for more details.

Note that Corollary 2.5 cannot be immediately applied in the setting of the bounded slope condition, since in general the non-linearity $g$ prevents affine functions from being variational sub- or super-solutions and thus from being admissible comparison functions. It is hence not possible, in this manner, to obtain an inequality of the form $a_{1} \leq u \leq a_{2}$ in $\Omega_{T}$ with $a_{1}, a_{2}$ affine functions. One way to deal with this problem is to assume that the Lagrangian is uniformly convex in a suitable sense. The strategy is then to bend the affine functions in space so that the divergence term dominates the lower order term $g$ in the Euler-Lagrange equation, causing the bent functions to be suitable barriers. This kind of ideas were previously used in the time-independent setting, see for example [28] and [8].

Definition 2.8. Let $\Phi:[0, \infty) \rightarrow[0, \infty)$ be a continuous function such that

$$
\lim _{s \rightarrow \infty} s \Phi(s)=+\infty .
$$

We say that $f \in C^{2}\left(\mathbb{R}^{n}\right)$ is $\Phi$-uniformly convex if

$$
D^{2} f(\eta) \xi \cdot \xi=\operatorname{tr}\left(D^{2} f(\eta) \xi \otimes \xi\right) \geq \Phi(|\eta|)|\xi|^{2}
$$

for all $\xi, \eta \in \mathbb{R}^{n}$. Here $\xi \otimes \xi$ denotes the $n \times n$ matrix whose $(i, j)$ entry is $\xi_{i} \xi_{j}$.

Note that this condition can be interpreted as a generalization of a coercivity condition imposed on the matrix $D^{2} f(\eta)$ or the bilinear form $(\zeta, \xi) \mapsto D^{2} f(\eta) \zeta \cdot \xi$, respectively. In particular, for a constant function $\Phi$, this condition reduces to a usual coercivity condition well-known from elliptic PDE theory and Definition 2.8 demands $f$ to be a strongly convex function.

Proposition 2.9. Suppose that the conditions (2.1) - (2.3) hold. Further suppose that $\Omega$ is convex, $\left(\mathrm{H}_{h, \omega}\right)$ holds with a Lipschitz modulus of continuity and that $f \in C^{2}\left(\mathbb{R}^{n}\right)$ is $\Phi$-uniformly convex. Suppose moreover that $u_{0}$ is Lipschitz in $\bar{\Omega}$ and $\left.u_{0}\right|_{\partial \Omega}$ satisfies the bounded slope condition with $Q>0$. Then there is $L>0$ such that a variational solution $u$ to the Cauchy-Dirichlet problem (1.1) satisfies

$$
|u(x, t)-u(y, t)| \leq L|x-y|
$$

whenever $(x, t),(y, t) \in \Omega_{T}$ are Lebesgue points of $u$. 


\section{Preliminary and auxiliary results}

In this section, we state some important results which will be used in our proofs in the later chapters. We start by proving both spatial and time localization principles. For the convenience of the reader, we will also give the proofs.

Lemma 3.1. (Spatial localization principle) Let $\tilde{\Omega} \subset \Omega \subset \mathbb{R}^{n}$ be bounded Lipschitz domains. Suppose $u: \Omega_{T} \rightarrow \mathbb{R}$ to be a variational sub-solution (resp. a variational super-solution) on $\Omega_{T}$, Then, $\left.u\right|_{\tilde{\Omega}_{T}}: \tilde{\Omega}_{T} \rightarrow \mathbb{R}$ is a variational sub-solution (resp. a variational super-solution) on $\tilde{\Omega}_{T}$.

Proof. We choose a function $\left.w \in u\right|_{\tilde{\Omega}_{T}}+L^{p}\left([0, T] ; W_{0}^{1, p}(\tilde{\Omega})\right) \cap L^{2}\left(\Omega_{T}\right)$ with $w \leq\left. u\right|_{\tilde{\Omega}_{T}}$ a.e. on $\tilde{\Omega}_{T}$ (resp. $w \geq\left. u\right|_{\tilde{\Omega}_{T}}$ a.e. on $\tilde{\Omega}_{T}$ ) and $\partial_{t} w \in L^{2}\left(\tilde{\Omega}_{T}\right)$. Observe that

$$
v:=\left\{\begin{array}{l}
w \text { in } \tilde{\Omega}_{T}, \\
u \text { in }(\Omega \backslash \tilde{\Omega})_{T},
\end{array}\right.
$$

is a valid test function in the inequality (2.5) of the variational sub-solution $u$ (resp. the variational super-solution $u$ ) on $\Omega_{T}$. Inserting this test function immediately leads to

$$
\begin{aligned}
\int_{\tilde{\Omega}_{T}} f(D u)+g(x, u) \mathrm{d} z \leq & \int_{\tilde{\Omega}_{T}} \partial_{t} w(w-u)+f(D w)+g(x, w) \mathrm{d} z \\
& -\left.\frac{1}{2} \int_{\tilde{\Omega}}(w-u)^{2}(x, \cdot) \mathrm{d} x\right|_{0} ^{T} .
\end{aligned}
$$

Since $w$ was chosen arbitrarily at the beginning, the last inequality proves that $\left.u\right|_{\tilde{\Omega}_{T}}$ is a variational sub-solution (resp. a variational super-solution) on $\tilde{\Omega}$, which concludes the proof.

Lemma 3.2. (Temporal localization principle) Let $\Omega \subset \mathbb{R}^{n}$ be a bounded Lipschitz domain, $0<t_{1}<t_{2}<T$, and define $\Omega_{t_{1}, t_{2}}:=\Omega \times\left(t_{1}, t_{2}\right)$. Suppose $u: \Omega_{T} \rightarrow \mathbb{R}$ to be a variational sub-solution (resp. a variational super-solution) on $\Omega_{T}$. Then, the restriction $\left.u\right|_{\Omega_{t_{1}, t_{2}}}$ is a variational sub-solution (resp. super-solution) on $\Omega_{t_{1}, t_{2}}$.

Proof. In the following, we will use the convenient abbreviation $u_{t_{1}, t_{2}}:=\left.u\right|_{\partial_{P} \Omega_{t_{1}, t_{2}}}$. We choose a function $w \in u+L^{p}\left(\left[t_{1}, t_{2}\right] ; W_{0}^{1, p}(\Omega)\right) \cap L^{2}\left(\Omega_{t_{1}, t_{2}}\right)$ with $w \leq\left. u\right|_{\Omega_{t_{1}, t_{2}}}$ a.e. in $\Omega_{t_{1}, t_{2}}$ (resp. $w \geq\left. u\right|_{\Omega_{t_{1}, t_{2}}}$ a.e. in $\left.\Omega_{t_{1}, t_{2}}\right), \partial_{t} w \in L^{2}\left(\Omega_{t_{1}, t_{2}}\right)$. Additionally, we define for $\vartheta \in\left(0, \frac{t_{2}-t_{1}}{2}\right)$ a cutoff function $\zeta_{\vartheta} \in W^{1, \infty}([0, T])$ with respect to time via

$$
\zeta_{\vartheta}(t):=\frac{t-t_{1}}{\vartheta} \mathbb{1}_{\left[t_{1}, t_{1}+\vartheta\right)}(t)+\mathbb{1}_{\left[t_{1}+\vartheta, t_{2}-\vartheta\right]}(t)+\frac{t_{2}-t}{\vartheta} \mathbb{1}_{\left(t_{2}-\vartheta, t_{2}\right]}(t)
$$

and set

$$
v:=\zeta_{\vartheta} w+\left(1-\zeta_{\vartheta}\right) u
$$


Since $v \in u+L^{p}\left([0, T] ; W_{0}^{1, p}(\Omega)\right) \cap L^{2}\left(\Omega_{T}\right)$ with $\partial_{t} v \in L^{2}\left(\Omega_{T}\right)$ and $v=$ $\zeta_{\vartheta} w+\left(1-\zeta_{\vartheta}\right) u \leq \zeta_{\vartheta} u+\left(1-\zeta_{\vartheta}\right) u=u$ (resp. $\left.v \geq u\right), v$ is a valid test function in the variational inequality (2.5). Therefore, testing (2.5) with $v$ first leads to

$$
\begin{aligned}
& \int_{\Omega_{T}} f(D u)+g(x, u) \mathrm{d} z \\
& \quad \leq \int_{\Omega_{T}} \zeta_{\vartheta}^{\prime} \zeta_{\vartheta}(w-u)^{2}+\zeta_{\vartheta}^{2} \partial_{t}(w-u)(w-u)+\zeta_{\vartheta} \partial_{t} u(w-u) \mathrm{d} z \\
& \quad+\int_{\Omega_{T}} f\left(\zeta_{\vartheta} D w+\left(1-\zeta_{\vartheta}\right) D u\right)+g\left(x, \zeta_{\vartheta} w+\left(1-\zeta_{\vartheta}\right) u\right) \mathrm{d} z,
\end{aligned}
$$

where the boundary term vanishes due to $\zeta_{\vartheta}(T)=\zeta_{\vartheta}(0)=0$.

In the following steps, we will split up the temporal region of integration $[0, T]$ into five distinct parts, given by the definition of the cut-off function $\zeta_{\vartheta}$. We will then analyze the behaviour of each integral as we send $\vartheta \downarrow 0$. To guarantee a good overview of this process, we will perform this procedure on each of the two integral terms above separately. We start with the integral involving $f$-and $g$-terms:

Due to convexity of both $\xi \mapsto f(\xi)$ as well as $u \mapsto g(x, u)$ for almost every $x \in \Omega$ and $\zeta_{\vartheta} \in[0,1]$, we have

$$
\begin{array}{rl}
\int_{\Omega_{T}} & f\left(\zeta_{\vartheta} D w+\left(1-\zeta_{\vartheta}\right) D u\right)+g\left(x, \zeta_{\vartheta} w+\left(1-\zeta_{\vartheta}\right) u\right) \mathrm{d} z \\
\leq & \int_{\Omega_{T}} \zeta_{\vartheta} f(D w)+\left(1-\zeta_{\vartheta}\right) f(D u)+\zeta_{\vartheta} g(x, w)+\left(1-\zeta_{\vartheta}\right) g(x, u) \mathrm{d} z \\
= & \int_{\Omega} \int_{0}^{t_{1}} f(D u)+g(x, u) \mathrm{d} t \mathrm{~d} x \\
& +\int_{\Omega} \int_{t_{1}}^{t_{1}+\vartheta} \frac{t-t_{1}}{\vartheta}(f(D w)+g(x, w)) \\
& +\left(1-\frac{t-t_{1}}{\vartheta}\right)(f(D u)+g(x, u)) \mathrm{d} t \mathrm{~d} x \\
& +\int_{\Omega} \int_{t_{1}+\vartheta}^{t_{2}-\vartheta} f(D w)+g(x, w) \mathrm{d} t \mathrm{~d} x \\
& +\int_{\Omega} \int_{t_{2}-\vartheta}^{t_{2}} \frac{t_{2}-t}{\vartheta}(f(D w)+g(x, w)) \\
& +\left(1-\frac{t_{2}-t}{\vartheta}\right)(f(D u)+g(x, u)) \mathrm{d} t \mathrm{~d} x \\
& +\int_{\Omega} \int_{t_{2}}^{T} f(D u)+g(x, u) \mathrm{d} t \mathrm{~d} x .
\end{array}
$$


Looking at the second integral when sending $\vartheta \downarrow 0$, we find using Lebesgue's differentiation theorem:

$$
\begin{aligned}
& \int_{t_{1}}^{t_{1}+\vartheta} \frac{t-t_{1}}{\vartheta}(f(D w)+g(x, w)) \mathrm{d} t \\
& =\int_{t_{1}}^{t_{1}+\vartheta}\left(t-t_{1}\right)(f(D w)+g(x, w)) \mathrm{d} t \longrightarrow 0 \\
& \int_{t_{1}}^{t_{1}+\vartheta}\left(1-\frac{t-t_{1}}{\vartheta}\right)(f(D u)+g(x, u)) \mathrm{d} t \\
& =\int_{t_{1}}^{t_{1}+\vartheta} f(D u)+g(x, u) \mathrm{d} t \\
& \quad-\int_{t_{1}}^{t_{1}+\vartheta}\left(t-t_{1}\right)(f(D u)+g(x, u)) \mathrm{d} t \longrightarrow 0 .
\end{aligned}
$$

Similarly, one can treat the fourth integral term and receive the same result. For the third term, we make the simple observation:

$$
\int_{t_{1}+\vartheta}^{t_{2}-\vartheta} f(D w)+g(x, w) \mathrm{d} t \longrightarrow \int_{t_{1}}^{t_{2}} f(D w)+g(x, w) \mathrm{d} t,
$$

as $\vartheta \downarrow 0$. Splitting up the time interval of the integral term of the term on the left-hand side of $(3.1)$ into $\left[0, t_{1}\right],\left[t_{1}, t_{2}\right]$, and $\left[t_{2}, T\right]$ and cancelling out the two integral terms appearing on either side, leaves us with the term

$$
\int_{\Omega_{t_{1}, t_{2}}} f(D u)+g(x, u) \mathrm{d} z
$$

remaining on the left-hand side of (3.1).

Now, we treat the integral terms involving time derivatives in (3.1). Using the product rule of differentiation backwards, inserting the term $\zeta_{\vartheta} \partial_{t} w(w-u)$, and integrating by parts, as well as splitting up the domain of integration the same way as before yield

$$
\begin{aligned}
\int_{\Omega_{T}} \zeta_{\vartheta}^{\prime} \zeta_{\vartheta}(w-u)^{2}+\zeta_{\vartheta}^{2} \partial_{t}(w-u)(w-u)+\zeta_{\vartheta} \partial_{t} u(w-u) \mathrm{d} z \\
=\int_{\Omega_{T}} \frac{1}{2} \partial_{t}\left(\zeta_{\vartheta}^{2}(w-u)^{2}\right)+\zeta_{\vartheta} \partial_{t} w(w-u)-\zeta_{\vartheta} \partial_{t}(w-u)(w-u) \mathrm{d} z \\
=\left.\frac{1}{2} \int_{\Omega} \zeta_{\vartheta}^{2}(\cdot)(w-u)^{2}(x, \cdot) \mathrm{d} x\right|_{0} ^{T}+\int_{\Omega_{T}} \zeta_{\vartheta} \partial_{t} w(w-u)-\frac{1}{2} \zeta_{\vartheta} \partial_{t}(w-u)^{2} \mathrm{~d} z \\
=\int_{\Omega_{T}} \zeta_{\vartheta} \partial_{t} w(w-u) \mathrm{d} z-\left.\frac{1}{2} \int_{\Omega} \zeta_{\vartheta}(\cdot)(w-u)^{2}(x, \cdot) \mathrm{d} x\right|_{0} ^{T}+\frac{1}{2} \int_{\Omega_{T}} \zeta_{\vartheta}^{\prime}(w-u)^{2} \mathrm{~d} z \\
=\int_{\Omega} \int_{t_{1}}^{t_{1}+\vartheta}\left(t-t_{1}\right) \partial_{t} w(w-u) \mathrm{d} t \mathrm{~d} x+\frac{1}{2} \int_{\Omega} f_{t_{1}}^{t_{1}+\vartheta}(w-u)^{2} \mathrm{~d} t \mathrm{~d} x \\
\quad+\int_{\Omega} \int_{t_{1}+\vartheta}^{t_{2}-\vartheta} \partial_{t} w(w-u) \mathrm{d} t \mathrm{~d} x
\end{aligned}
$$




$$
+\int_{\Omega} \int_{t_{2}-\vartheta}^{t_{2}}\left(t_{2}-t\right) \partial_{t} w(w-u) \mathrm{d} t \mathrm{~d} x-\frac{1}{2} \int_{\Omega} f_{t_{2}-\vartheta}^{t_{2}}(w-u)^{2} \mathrm{~d} t \mathrm{~d} x .
$$

Sending $\vartheta \downarrow 0$ and using Lebesgue's differentiation theorem results in

$$
\begin{gathered}
\int_{t_{1}+\vartheta}^{t_{2}-\vartheta} \partial_{t} w(w-u) \mathrm{d} t \longrightarrow \int_{t_{1}}^{t_{2}} \partial_{t} w(w-u) \mathrm{d} t \\
\int_{t_{1}}^{t_{1}+\vartheta}\left(t-t_{1}\right) \partial_{t} w(w-u) \mathrm{d} t \longrightarrow 0 \\
f_{t_{1}}^{t_{1}+\vartheta}(w-u)^{2} \mathrm{~d} t \longrightarrow(w-u)\left(\cdot, t_{1}\right)
\end{gathered}
$$

which implies the last sum of integral terms to converge to

$$
\int_{\Omega_{t_{1}, t_{2}}} \partial_{t} w(w-u) \mathrm{d} z-\left.\frac{1}{2} \int_{\Omega}(w-u)^{2}(x, \cdot) \mathrm{d} x\right|_{t_{1}} ^{t_{2}} .
$$

Finally, collecting all remaining terms on both sides of (3.1), we are left with

$$
\begin{aligned}
\int_{\Omega_{t_{1}, t_{2}}} f(D u)+g(x, u) \mathrm{d} z \leq & \int_{\Omega_{t_{1}, t_{2}}} \partial_{t} w(w-u)+f(D w)+g(x, w) \mathrm{d} z \\
& -\left.\frac{1}{2} \int_{\Omega}(w-u)^{2}(x, \cdot) \mathrm{d} x\right|_{t_{1}} ^{t_{2}} .
\end{aligned}
$$

Since $w$ was chosen arbitrarily, we have proven that $\left.u\right|_{\Omega_{t_{1}, t_{2}}}$ is a variational subsolution (resp. supersolution) in $\Omega_{t_{1}, t_{2}}$ which finishes the proof.

Lemma 3.3. (Comparison Principle) Let $u$ and $\tilde{u}$ be a variational sub-solution and a super-solution on $\Omega_{T}$, respectively. Suppose that $u(\cdot, 0) \leq \tilde{u}(\cdot, 0)$ almost everywhere in $\Omega$ and that

$$
u(\cdot, t) \leq \tilde{u}(\cdot, t) \text { on } \partial \Omega
$$

in the sense of traces for almost all $t \in(0, T)$. Then $u \leq \tilde{u}$ a.e. in $\Omega_{T}$.

Proof. Let $\tau \in(0, T)$. Define the test functions

$$
v:=\min (u, \tilde{u}) \text { and } w:=\max (u, \tilde{u}) .
$$

By Lemma 3.2, $v$ and $w$ are admissible test functions for $u$ and $\tilde{u}$ in their respective variational inequality (2.5) in $\Omega_{\tau}$. Adding up the variational inequalities, we obtain

$$
\begin{aligned}
0 \leq & \int_{\Omega_{\tau}} \partial_{t} v(v-u)+\partial_{t} w(w-\tilde{u}) \mathrm{d} z \\
& +\int_{\Omega_{\tau}} g(x, v)-g(x, u)+g(x, w)-g(x, \tilde{u}) \mathrm{d} z \\
& +\int_{\Omega_{\tau}} f(D v)-f(D u)+f(D w)-f(D \tilde{u}) \mathrm{d} z \\
& -\frac{1}{2} \int_{\Omega}(v-u)^{2}+\left.(w-\tilde{u})^{2} \mathrm{~d} x\right|_{0} ^{\tau} .
\end{aligned}
$$


Observe that

$$
\begin{aligned}
& g(x, v)-g(x, u)+g(x, w)-g(x, \tilde{u}) \\
& \quad=\mathbb{1}_{\{u<\tilde{u}\}}(g(x, u)-g(x, u)+g(x, \tilde{u})-g(x, \tilde{u})) \\
& \quad+\mathbb{1}_{\{\tilde{u} \leq u\}}(g(x, \tilde{u})-g(x, u)+g(x, u)-g(x, \tilde{u})) \\
& \quad=0 .
\end{aligned}
$$

Similarly,

$$
f(D v)-f(D u)+f(D w)-f(D \tilde{u})=0 .
$$

Using the last two displays and the identities $v-u=-(u-\tilde{u})_{+}$and $w-\tilde{u}=$ $(u-\tilde{u})_{+}$, we obtain from (3.2)

$$
\begin{aligned}
\left.\int_{\Omega}(u-\tilde{u})_{+}^{2} \mathrm{~d} x\right|_{0} ^{\tau} & \leq \int_{\Omega_{\tau}} \partial_{t}(w-v)(u-\tilde{u})_{+} \mathrm{d} z=\int_{\Omega_{\tau}} \partial_{t}|u-\tilde{u}|(u-\tilde{u})_{+} \mathrm{d} z \\
& =\int_{\Omega_{\tau}} \frac{1}{2} \partial_{t}(u-\tilde{u})_{+}^{2} \mathrm{~d} z=\left.\frac{1}{2} \int_{\Omega}(u-\tilde{u})_{+}^{2} \mathrm{~d} x\right|_{0} ^{\tau} .
\end{aligned}
$$

Since $(u(\cdot, 0)-\tilde{u}(\cdot, 0))_{+}=0$ in $\Omega$, the above yields

$$
\frac{1}{2} \int_{\Omega}(u(\cdot, \tau)-\tilde{u}(\cdot, \tau))_{+}^{2} \mathrm{~d} x \leq 0 .
$$

Thus $u(\cdot, \tau) \leq \tilde{u}(\cdot, \tau)$ almost everywhere in $\Omega$. Since $\tau \in(0, T)$ was arbitrary, we obtain that $u \leq \tilde{u}$ a.e. in $\Omega_{T}$.

\section{Shifted sub- and super-solutions}

In this section we show that suitably shifted and lifted variational super-solutions are still super-solutions on the intersection of the original domain and the shifted domain. We begin with the following simple observation.

Proposition 4.1. Let $\omega$ be a modulus of continuity. Suppose that $g$ fulfils (2.2) and $\left(\mathrm{H}_{h, \omega}\right)$ for any $h \in \mathbb{R}^{n}$. Suppose further that $w: \Omega_{T} \rightarrow \mathbb{R}$ is bounded. Then $(x, t) \mapsto g_{u}^{+}(x, w(x, t))$ is bounded in $\Omega_{T}$.

Proof. Denote $M:=\|w\|_{L^{\infty}\left(\Omega_{T}\right)}<\infty$ and fix $x_{0} \in \Omega$. Let $x \in \Omega$. Recall that the condition $\left(\mathrm{H}_{h, \omega}\right)$ states that

$v \geq u+\omega(|h|) \Longrightarrow g_{u}^{+}(y-h, v) \geq g_{u}^{+}(y, u)$ for all $h, y \in \mathbb{R}^{n}$ and $v, u \in \mathbb{R}$.

Using the convexity of $g$, in particular that $g_{u}^{+}$is monotonically non-decreasing, and applying the above with $v:=-M, u:=-M-\omega(|h|), y:=x_{0}, h:=x_{0}-x$, we obtain

$$
\begin{aligned}
g_{u}^{+}(x, w(x, t)) & \geq g_{u}^{+}(x,-M) \geq g_{u}^{+}\left(x_{0},-M-\omega\left(\left|x_{0}-x\right|\right)\right) \\
& \geq g_{u}^{+}\left(x_{0},-M-\omega(\operatorname{diam} \Omega)\right) .
\end{aligned}
$$

The upper bound is obtained analogously and thus $(x, t) \mapsto g_{u}^{+}(x, w(x, t))$ is bounded. 
Theorem 4.2. Suppose that $h \in \mathbb{R}^{n}, \Omega \cap \Omega_{h}$ is Lipschitz and that $\omega$ is a modulus of continuity. Let the assumptions (2.1) - (2.3) and $\left(\mathrm{H}_{h, \omega}\right)$ hold. Let $u$ be a bounded variational super-solution on $\Omega_{T}$. Then

$$
\tilde{u}(x, t):=u_{-h}(x, t)+\omega(|h|)
$$

is a variational super-solution on $\left(\Omega \cap \Omega_{-h}\right)_{T}$. Similarly, for a bounded variational sub-solution $w$ on $\Omega_{T}$, the function

$$
\tilde{w}(x, t):=w_{h}(x, t)-\omega(|h|)
$$

is a variational sub-solution on $\left(\Omega \cap \Omega_{h}\right)_{T}$.

Here, $\Omega_{h}$ and $\Omega_{-h}$ denote the shifted sets and $u_{-h}, u_{h}$ the shifted functions as defined in (2.6).

Proof. (Step 1) Denote $c:=\omega(|h|)>0$ and $\tilde{\Omega}:=\Omega \cap \Omega_{-h}$. Let $(x, t) \in \tilde{\Omega}_{T}$. By convexity of $g$,

$$
g(x, \tilde{u}(x, t)) \leq g(x, u(x, t))+(\tilde{u}(x, t)-u(x, t)) g_{u}^{+}(x, \tilde{u}(x, t)) .
$$

Since $u$ and $\tilde{u}$ are bounded, it therefore follows from Proposition 4.1 that

$$
\int_{\tilde{\Omega}_{T}} g(x, \tilde{u}) \mathrm{d} z \leq \int_{\tilde{\Omega}_{T}} g(x, u) \mathrm{d} z+\int_{\tilde{\Omega}_{T}}|u-\tilde{u}|\left|g_{u}^{+}(x, \tilde{u})\right| \mathrm{d} z<\infty .
$$

Consequently we have

$$
\int_{\tilde{\Omega}_{T}} g(x, \tilde{u})+f(D \tilde{u}) \mathrm{d} z<\infty
$$

as required in the definition of variational super-solutions.

(Step 2) Let $\tilde{v} \in \tilde{u}+L^{p}\left([0, T] ; W_{0}^{1, p}(\tilde{\Omega})\right) \cap L^{2}\left(\tilde{\Omega}_{T}\right)$ be such that $\tilde{v} \geq \tilde{u}$ in $\tilde{\Omega}$ and $\partial_{t} \tilde{v} \in L^{2}\left(\tilde{\Omega}_{T}\right)$. We need to show that $\tilde{u}$ satisfies the variational inequality (2.5) with respect to the test function $\tilde{v}$. We may suppose that

$$
\int_{\tilde{\Omega}_{T}} f(D \tilde{v})+g(x, \tilde{v}) \mathrm{d} z<\infty
$$

as otherwise the variational inequality holds trivially. We set

$$
v(x, t):= \begin{cases}\tilde{v}_{h}(x, t)-c, & x \in \tilde{\Omega}_{h}, \\ u(x, t), & x \in \Omega \backslash \tilde{\Omega}_{h} .\end{cases}
$$

Then $v \in u+L^{p}\left([0, T] ; W_{0}^{1, p}(\Omega)\right) \cap L^{2}\left(\Omega_{T}\right)$ with $v \geq u$ in $\Omega_{T}$. Using $v$ as a test function for $u$ in the variational inequality (2.5), we obtain 


$$
\begin{aligned}
0 \leq & \int_{\Omega_{T}} \partial_{t} v(v-u)+f(D v)-f(D u) \mathrm{d} z-\left.\frac{1}{2} \int_{\Omega}(v-u)^{2} \mathrm{~d} x\right|_{0} ^{T} \\
& +\int_{\Omega_{T}} g(x, v)-g(x, u) \mathrm{d} z \\
= & \int_{\tilde{\Omega}_{h, T}} \partial_{t} \tilde{v}_{h}\left(\tilde{v}_{h}-(u+c)\right)+f\left(D \tilde{v}_{h}\right)-f(D u) \mathrm{d} z-\left.\frac{1}{2} \int_{\tilde{\Omega}_{h}}\left(\tilde{v}_{h}-(u+c)\right)^{2} \mathrm{~d} x\right|_{0} ^{T} \\
& +\int_{\tilde{\Omega}_{h, T}} g\left(x, \tilde{v}_{h}-c\right)-g(x, u) \mathrm{d} z \\
= & \int_{\tilde{\Omega}_{T}} \partial_{t} \tilde{v}\left(\tilde{v}-\left(u_{-h}+c\right)\right)+f(D \tilde{v})-f\left(D u_{-h}\right) \mathrm{d} z-\left.\frac{1}{2} \int_{\tilde{\Omega}}\left(\tilde{v}-\left(u_{-h}+c\right)\right)^{2} \mathrm{~d} x\right|_{0} ^{T} \\
& +\int_{\tilde{\Omega}_{h, T}} g\left(x, \tilde{v}_{h}-c\right)-g(x, u) \mathrm{d} z \\
= & \int_{\tilde{\Omega}_{T}} \partial_{t} \tilde{v}(\tilde{v}-\tilde{u})+f(D \tilde{v})-f(D \tilde{u})+g(x, \tilde{v})-g(x, \tilde{u}) \mathrm{d} z-\left.\frac{1}{2} \int_{\tilde{\Omega}}(\tilde{v}-\tilde{u})^{2} \mathrm{~d} x\right|_{0} ^{T} \\
& +\int_{\tilde{\Omega}_{h, T}} g\left(x, \tilde{v}_{h}-c\right)-g(x, u) \mathrm{d} z-\int_{\tilde{\Omega}_{T}} g(x, \tilde{v})-g(x, \tilde{u}) \mathrm{d} z .
\end{aligned}
$$

If we can now show that the last two integrals together are non-positive, we have established that $\tilde{u}$ satisfies the variational inequality with respect to $\tilde{v}$. This would imply that $\tilde{u}$ is a variational super-solution on $\tilde{\Omega}_{T}$, as desired. To this end, we first observe that

$$
\int_{\tilde{\Omega}_{T}} g(x, \tilde{v})-g(x, \tilde{u}) \mathrm{d} z=\int_{\tilde{\Omega}_{h, T}} g\left(x-h, \tilde{v}_{h}\right)-g(x-h, u+c) \mathrm{d} z .
$$

In the following, we omit the time point $t$. Observe that the previous equation together with (4.1) guarantees also that $\int_{\tilde{\Omega}_{h, T}} g(x, v) \mathrm{d} z<\infty$. Consequently, it suffices to prove the following inequality:

$$
\begin{aligned}
& g(x-h, \tilde{v}(x-h))-g(x-h, u(x)+c) \\
& \quad \geq g(x, \tilde{v}(x-h)-c)-g(x, u(x)) \text { a.e. in } \tilde{\Omega}_{h, T} .
\end{aligned}
$$

To this end, fix $(x, t) \in \tilde{\Omega}_{h, T}$ for which the above quantities are defined and finite. Set

$$
\psi(y):=g(x-h, y) \text { and } \phi(y):=g(x, y-c)
$$

for $y \in[u(x)+c, \tilde{v}(x-h)]$. We remark that this interval is never empty since $\tilde{v} \geq \tilde{u}$ in $\tilde{\Omega}_{T}$ implies that

$$
\tilde{v}(x-h) \geq \tilde{u}(x-h)=u_{-h}(x-h)+c=u(x)+c .
$$

In particular, if it is a singleton, both sides of (4.2) are zero. Therefore we are left with the case where $u(x)+c<\tilde{v}(x-h)$. Then $\psi$ and $\phi$ have right-derivatives

$$
\psi_{+}^{\prime}(y)=g_{u}^{+}(x-h, y) \text { and } \phi_{+}^{\prime}(y)=g_{u}^{+}(x, y-c),
$$


where $g_{u}^{+}$denotes the right-derivative of $g$ with respect to the second variable. We insert $c=\omega(|h|)$, which allows us to use the assumption $\left(H_{h, \omega}\right)$ to obtain

$$
\psi_{+}^{\prime}(y)=g_{u}^{+}(x-h, y) \geq g_{u}^{+}(x, y-c)=\phi_{+}^{\prime}(y)
$$

in $[u(x)+c, \tilde{v}(x-h)]$. Thus, integration over this interval yields

$$
\psi(\tilde{v}(x-h))-\psi(u(x)+c) \geq \phi(\tilde{v}(x-h))-\phi(u(x)+c)),
$$

which is exactly (4.2). Thus $\tilde{u}$ fulfills the variational inequality and is a variational super-solution. To obtain the respective result for sub-solutions, the calculations are similar. In the assumption $\left(\mathrm{H}_{h, \omega}\right)$ one can then replace $x$ with $x+h$ and set $v=y+c, u=c$ to come to the analogous conclusion.

\section{Proof of the main result}

This section is dedicated to proving the main result (Theorem 2.4) of this article. We begin with a technical lemma which says that the Haar-Rado type condition (2.7) implies that

$$
u_{h}(\cdot, t)-\omega(|h|) \leq u(\cdot, t) \quad \text { on } \partial(\Omega \cap \Omega)_{h}
$$

in the sense of traces for any $h \in \mathbb{R}^{n}$. Since the shifted function at the left-hand side is still a variational sub-solution by Theorem 4.2, while the boundedness will be shown in Lemma 5.2, it follows from the comparison principle that the inequality holds almost everywhere in $\Omega \cap \Omega_{h}$. Writing $h=x-y$ then essentially yields $u(x, t)-u(y, t) \leq \omega(|x-y|)$ whenever $(x, t)$ and $(y, t)$ are Lebesgue points of $u$.

Lemma 5.1. Let $\partial\left(\Omega \cap \Omega_{h}\right)$ be Lipschitz. Suppose that $u_{0} \in W^{1, p}(\Omega)$ is $\omega$ continuous on $\bar{\Omega}$ and let

$$
u \in u_{0}+L^{p}\left([0, T] ; W_{0}^{1, p}(\Omega)\right) \cap L^{2}\left(\Omega_{T}\right) \text { with } \partial_{t} u \in L^{2}\left(\Omega_{T}\right) .
$$

Suppose moreover that

$$
\left|u(x, t)-u_{0}(\gamma)\right| \leq \omega(|x-\gamma|) \quad \text { for a.e. }(x, t) \in \Omega_{T} \text { and } \gamma \in \partial \Omega .
$$

Then

$$
u_{h}(\cdot, t)-\omega(|h|) \leq u(\cdot, t) \quad \text { on } \partial\left(\Omega \cap \Omega_{h}\right)
$$

in the sense of traces for almost all $t \in(0, T)$.

Proof. For the properties of the trace that we use in this proof, we once again refer to [26, Section 3]. We proceed similar as in the proof for [26, Lemma 5.2]. Fix $t \in(0, T)$ such that $u(\cdot, t)=u_{0}(\cdot)$ on $\partial \Omega$ in the sense of traces. We wish to show that

$$
\lim _{r \rightarrow 0} f_{B_{r}(\gamma) \cap \Omega \cap \Omega_{h}} u_{h}(x, t)-u(x, t) \mathrm{d} x \leq \omega(|h|)
$$


for $\mathcal{H}^{n-1}$-almost every $\gamma \in \partial\left(\Omega \cap \Omega_{h}\right)$. To this end, let

$$
\gamma \in \partial\left(\Omega \cap \Omega_{h}\right) \subset\left(\partial \Omega \cap \Omega_{h}\right) \cup\left(\partial \Omega_{h} \cap \Omega\right) \cup\left(\partial \Omega \cap \partial \Omega_{h}\right) .
$$

First case: $\gamma \in \partial \Omega \cap \boldsymbol{\Omega}_{\boldsymbol{h}}$. Let $r>0$ be so small that $B_{r}(\gamma) \subset \Omega_{h}$. Then

$$
\begin{aligned}
f_{B_{r}(\gamma) \cap \Omega} u_{h}(x, t)-u(x, t) \mathrm{d} x= & f_{B_{r}(\gamma) \cap \Omega} u_{h}(x, t)-u_{0}(\gamma) \mathrm{d} x \\
& +f_{B_{r}(\gamma) \cap \Omega} u_{0}(\gamma)-u(x, t) \mathrm{d} x \\
= & f_{B_{r}(\gamma) \cap \Omega} u(x-h, t)-u_{0}(\gamma) \mathrm{d} x \\
& +f_{B_{r}(\gamma) \cap \Omega} u_{0}(\gamma)-u(x, t) \mathrm{d} x \\
\leq & f_{B_{r}(\gamma) \cap \Omega} \omega(|x-h-\gamma|) \mathrm{d} x \\
& +f_{B_{r}(\gamma) \cap \Omega} u_{0}(\gamma)-u(x, t) \mathrm{d} x,
\end{aligned}
$$

where the last inequality follows from (5.1). Since $\omega$ is continuous, the first integral at the right-hand side converges to $\omega(|h|)$ as $r \rightarrow 0$. The second integral on the other hand converges to zero for $\mathcal{H}^{n-1}$-almost every $\gamma \in \partial \Omega$. Thus we obtain (5.2).

Second case: $\boldsymbol{\gamma} \in \partial \boldsymbol{\Omega}_{\boldsymbol{h}} \cap \boldsymbol{\Omega}$. Let $r>0$ be so small that $B_{r}(\gamma) \subset \Omega$. Then

$$
\begin{aligned}
& f_{B_{r}(\gamma) \cap \Omega_{h}} u_{h}(x, t)-u(x, t) \mathrm{d} x \\
& =f_{B_{r}(\gamma-h) \cap \Omega} u(x, t)-u(x+h, t) \mathrm{d} x \\
& =f_{B_{r}(\gamma-h) \cap \Omega} u(x, t)-u_{0}(\gamma-h) \mathrm{d} x \\
& \quad+f_{B_{r}(\gamma-h) \cap \Omega} u_{0}(\gamma-h)-u(x+h, t) \mathrm{d} x \\
& \leq f_{B_{r}(\gamma-h) \cap \Omega} u(x, t)-u_{0}(\gamma-h) \mathrm{d} x \\
& \quad+f_{B_{r}(\gamma-h) \cap \Omega} \omega(|x+h-(\gamma-h)|) \mathrm{d} x,
\end{aligned}
$$

where the last inequality follows from (5.1) since $\gamma-h \in \partial \Omega$. As $r \rightarrow 0$, the first integral at the right-hand side converges to zero for $\mathcal{H}^{n-1}$-almost every $\gamma \in \partial \Omega$ and the second integral converges to $\omega(|h|)$. Thus we obtain (5.2).

Third case: $\boldsymbol{\gamma} \in \partial \boldsymbol{\Omega} \cap \partial \boldsymbol{\Omega}_{\boldsymbol{h}} \cap \partial\left(\boldsymbol{\Omega} \cap \boldsymbol{\Omega}_{\boldsymbol{h}}\right)$. By [26, Lemma 3.1] for $\mathcal{H}^{n-1}$-almost every $\gamma \in \partial \Omega \cap \partial \Omega_{h} \cap \partial\left(\Omega \cap \Omega_{h}\right)$ it holds that

$$
\begin{gathered}
\operatorname{tr}^{\Omega} u(\gamma, t)=\operatorname{tr}^{\Omega \cap \Omega_{h}} u(\gamma, t)=u_{0}(\gamma), \\
\operatorname{tr}^{\Omega_{h}} u(\gamma-h, t)=\operatorname{tr}^{\Omega \cap \Omega_{h}} u(\gamma-h, t)=u_{0}(\gamma-h) .
\end{gathered}
$$


We have

$$
\begin{aligned}
f_{B_{r}(\gamma) \cap \Omega \cap \Omega_{h}} u_{h}(x, t)-u(x, t) \mathrm{d} x= & f_{B_{r}(\gamma) \cap \Omega \cap \Omega_{h}} u(x-h, t)-u_{0}(\gamma-h) \mathrm{d} x \\
& +f_{B_{r}(\gamma) \cap \Omega \cap \Omega_{h}} u_{0}(\gamma)-u(x, t) \mathrm{d} x \\
& +f_{B_{r}(\gamma) \cap \Omega \cap \Omega_{h}} u_{0}(\gamma-h)-u_{0}(\gamma) \mathrm{d} x .
\end{aligned}
$$

As $r \rightarrow 0$, the first two integrals on the right-hand side converge to zero by (5.3). Moreover, we have $u_{0}(\gamma-h)-u_{0}(\gamma) \leq \omega(|h|)$ by $\omega$-continuity. Thus we obtain (5.2).

Lemma 5.2. Suppose the conditions (2.1) - (2.3) are in force. Further suppose that $u$ is a variational solution to the Cauchy-Dirichlet problem (1.1) on $\Omega_{T}$ and that $u_{0}$ is bounded in $\bar{\Omega}$. Then $u$ is bounded in $\Omega_{T}$.

Proof. We only show that $u$ is bounded from above as a lower bound follows analogously. Let $M:=\sup _{x \in \bar{\Omega}}\left|u_{0}(x)\right|$. We show that for large enough $\beta>0$, the function

$$
a(x, t):=M+\beta t
$$

is a variational super-solution on $\Omega_{T}$. Since $a \geq u$ on $\partial_{P} \Omega_{T}$, it follows from the comparison principle (Lemma 3.3) that

$$
u \leq a \leq M+\beta T \text { a.e. in } \Omega_{T}
$$

It now remains to show that $a$ is a variational super-solution on $\Omega_{T}$. To this aim, let $v \in a+L^{p}\left([0, T] ; W_{0}^{1, p}\left(\Omega_{T}\right)\right) \cap L^{2}\left(\Omega_{T}\right), v \geq a$ with $\partial_{t} v \in L^{2}\left(\Omega_{T}\right)$ be a test function. By convexity of $f$ for any $\xi \in \mathbb{R}^{n}$ there is $\lambda \xi \in \mathbb{R}^{n}$ such that

$$
f(\xi+\zeta) \geq f(\xi)+\lambda \xi \cdot \zeta .
$$

We define $\varphi(x, t):=v(x, t)-a(x, t)$ and use the above with $\xi:=D a(x, t)=0$ and $\zeta(x, t):=D \varphi(x, t)$ to obtain

$$
f(D v)=f(D a+D \varphi) \geq f(D a)+\lambda \cdot D \varphi .
$$

Integrating this over $\Omega_{T}$ we get

$$
\int_{\Omega_{T}} f(D v) \mathrm{d} z \geq \int_{\Omega_{T}} f(D a)+\lambda \cdot D \varphi \mathrm{d} z=\int_{\Omega_{T}} f(D a) \mathrm{d} z,
$$

where the last identity follows from the Gauss-Green theorem since $\varphi(\cdot, t) \in$ $W_{0}^{1, p}(\Omega)$ for almost all $t$ (cf. [13, Theorem 4.6]). Observe then that

$$
\partial_{t} v(v-a)=\frac{1}{2} \partial_{t}(v-a)^{2}+\partial_{t} a(v-a)
$$


Thus

$$
\begin{aligned}
& \int_{\Omega_{T}} \partial_{t} v(v-a)+g(x, v)-g(x, a) \mathrm{d} z-\left.\frac{1}{2} \int_{\Omega_{T}}(v-a)^{2}(x, \cdot) \mathrm{d} x\right|_{0} ^{T} \\
& =\int_{\Omega_{T}} \frac{1}{2} \partial_{t}(v-a)^{2}+\partial_{t} a(v-a)+g(x, v) \\
& \quad-g(x, a) \mathrm{d} z-\left.\frac{1}{2} \int_{\Omega}(v-a)^{2}(x, \cdot) \mathrm{d} x\right|_{0} ^{T} \\
& \quad \geq \int_{\Omega_{T}} \partial_{t} a(v-a)+(v-a) g_{u}^{+}(a, x) \mathrm{d} z,
\end{aligned}
$$

where in the last inequality we also used the convexity of $g(x, \cdot)$. In the view of (5.4) and (5.5) it now suffices to show that

$$
\partial_{t} a(v-a)+(v-a) g_{u}^{+}(a, x)=\left(\beta+g_{u}^{+}(M+\beta t, x)\right)(v-a) \geq 0 .
$$

Since $v-a \geq 0$ and by convexity $g_{u}^{+}(M+\beta t) \geq g_{u}^{+}(M)$, this follows by taking $\beta \geq\left|g_{u}^{+}(M)\right|$.

We are now ready to finish the proof of our main theorem.

Proof of Theorem 2.4. Fix $h \in \mathbb{R}^{n}$. Then by Lemma 5.1 we have

$$
u_{h}(\cdot, t)-\omega(|h|) \leq u(\cdot, t) \quad \text { on } \partial\left(\Omega \cap \Omega_{h}\right)
$$

in the sense of traces for almost all $t \in(0, T)$. Moreover, by $\omega$-continuity of $u_{0}$ we have

$$
u_{h}(\cdot, 0)-\omega(|h|) \leq u(\cdot, 0) \text { in } \overline{\Omega \cap \Omega_{h}} .
$$

Since $u$ is bounded in $\Omega_{T}$ by Lemma 5.2, it follows from Theorem 4.2 that the function $u_{h}-\omega(|h|)$ is a variational sub-solution on $\left(\Omega \cap \Omega_{h}\right)_{T}$. Thus the comparison principle (Lemma 3.3) implies that

$$
u_{h}-\omega \leq u \text { a.e. in }\left(\Omega \cap \Omega_{h}\right)_{T} .
$$

Let $\left(x_{0}, t_{0}\right),\left(y_{0}, t_{0}\right) \in \Omega_{T}$ be Lebesgue points of $u$. Take $r>0$ so small that both $Q_{r}\left(x_{0}, t_{0}\right), Q_{r}\left(y_{0}, t_{0}\right) \subset \Omega_{T}$. Let $h=x_{0}-y_{0}$. Then we have $Q_{r}\left(x_{0}, t_{0}\right) \subset$ $\left(\Omega \cap \Omega_{h}\right)_{T}$. Thus

$$
f_{Q_{r}\left(x_{0}, t_{0}\right)} u_{h}-u \mathrm{~d} z \leq f_{Q_{r}\left(x_{0}, t_{0}\right)} \omega(|h|) \mathrm{d} z,
$$

that is,

$$
\begin{aligned}
f_{Q_{r}\left(x_{0}, t_{0}\right)} \omega\left(\left|x_{0}-y_{0}\right|\right) \mathrm{d} z & \geq f_{Q_{r}\left(x_{0}, t_{0}\right)} u\left(x-x_{0}+y_{0}, t\right)-u(x, t) \mathrm{d} z \\
& =f_{Q_{r}\left(y_{0}, t_{0}\right)} u(x, t) \mathrm{d} z-f_{Q_{r}\left(x_{0}, t_{0}\right)} u(x, t) \mathrm{d} z .
\end{aligned}
$$

Letting $r \rightarrow 0$, this implies that $\omega\left(\left|x_{0}-y_{0}\right|\right) \geq u\left(y_{0}, t_{0}\right)-u\left(x_{0}, t_{0}\right)$. 
Proof of Corollary 2.5. For $(x, t) \in \Omega_{T}$ and $\gamma \in \partial \Omega$, we have

$$
u(x, t)-u_{0}(\gamma) \leq l_{2}(x)-u_{0}(\gamma)=l_{2}(x)-l_{2}(\gamma) \leq \omega(|x-\gamma|) .
$$

Similarly,

$$
u(x, t)-u_{0}(\gamma) \geq l_{1}(x)-u_{0}(\gamma)=l_{1}(x)-l_{1}(\gamma) \geq-\omega(|x-\gamma|) .
$$

Thus

$$
\left|u(x, t)-u_{0}(\gamma)\right| \leq \omega(|x-\gamma|) \text { for a.e. }(x, t) \in \Omega_{T} \text { and } \gamma \in \partial \Omega .
$$

Thus we can apply Theorem 2.4, which shows the claim.

\section{An application with the bounded slope condition}

In this section we apply the Haar-Rado type theorem to obtain the spatial Lipschitz continuity of variational solutions, provided that $f \in C^{2}\left(\mathbb{R}^{n}\right)$ is $\Phi$-uniformly convex and $u_{0}$ satisfies the bounded slope condition. We begin with the following lemma, which says that affine functions can be bent so that they become suitable barriers. The definition of the barrier function is in the spirit of Bousquet and Brasco [8, Proposition 4.2].

Lemma 6.1. Let $g$ be as in (2.2) and $\left(\mathrm{H}_{h, \omega}\right)$. Suppose that $\Omega$ is convex and that $f \in C^{2}\left(\mathbb{R}^{n}\right)$ is uniformly $\Phi$-convex. Let $x_{0} \in \partial \Omega$ and let $a: \mathbb{R}^{n} \rightarrow \mathbb{R}$ be an affine function. Then there is $\beta>0$ and $\eta \in \mathbb{R}^{n},|\eta|=1$, such that

$$
\widehat{a}(x):=a(x)+\beta\left(x-x_{0}\right) \cdot \eta\left(3 \operatorname{diam} \Omega-\left(x-x_{0}\right) \cdot \eta\right),
$$

is a variational super-solution in $\Omega_{T}$. Moreover, $\widehat{a} \geq a$ in $\bar{\Omega}$.

Proof. Since $\Omega$ is convex, we can rotate and translate it so that $x_{0}=0$ and $\Omega \subset$ $\left\{x \in \mathbb{R}^{n}: x_{1}>0\right\}$. We set

$$
\widehat{a}(x):=a(x)+\beta x_{1}\left(3 \operatorname{diam} \Omega-x_{1}\right) .
$$

Let $v \geq \widehat{a}$ be a test function for the variational inequality. To see that $\widehat{a}$ is a variational super-solution, we need to show that

$$
\begin{aligned}
& \int_{\Omega_{T}} f(D v)-f(D \widehat{a}) \mathrm{d} z \\
& \quad+\int_{\Omega_{T}} \partial_{t} v(v-\widehat{a})+g(x, v)-g(x, \widehat{a}) \mathrm{d} z \\
& \quad-\left.\frac{1}{2} \int_{\Omega}(v-\widehat{a})^{2}(x, \cdot) \mathrm{d} x\right|_{0} ^{T} \geq 0 .
\end{aligned}
$$

Since $\partial_{t} \widehat{a}=0$, we have

$$
\partial_{t} v(v-\widehat{a})=\frac{1}{2} \partial_{t}(v-\widehat{a})^{2},
$$


whence

$$
\begin{aligned}
& \int_{\Omega_{T}} \partial_{t} v(v-\widehat{a})+g(x, v)-g(x, \widehat{a}) \mathrm{d} z-\left.\frac{1}{2} \int_{\Omega}(v-\widehat{a})^{2}(x, \cdot) \mathrm{d} x\right|_{0} ^{T} \\
& \quad=\int_{\Omega_{T}} g(x, v)-g(x, \widehat{a}) \mathrm{d} z .
\end{aligned}
$$

Furthermore, since $\widehat{a} \leq v$, it follows from convexity of $g(x, \cdot)$ that

$$
g(x, v)-g(x, \widehat{a}) \geq(v-\widehat{a}) g_{u}^{+}(x, \widehat{a}) \quad \text { almost everywhere in } \Omega_{T} .
$$

Thus in the view of (6.1), (6.2) and (6.3) it suffices to show that

$$
\int_{\Omega} f(D v)-f(D \widehat{a})+(v-\widehat{a}) g_{u}^{+}(x, \widehat{a}) \mathrm{d} z \geq 0 .
$$

Since $f$ is $C^{2}$ and convex, for any $\xi \in \mathbb{R}^{n}$ we have

$$
f(\xi+\zeta) \geq f(\xi)+\zeta \cdot D f(\xi) \text { for all } \zeta \in \mathbb{R}^{n}
$$

We set $\varphi(x, t):=v(x, t)-\widehat{a}(x, t)$ and apply the above with $\xi:=D \widehat{a}(x)$ and $\zeta:=D \varphi(x, t)$. We obtain

$$
\begin{aligned}
f(D \widehat{a}(x)+D \varphi(x, t)) & =f(\xi+\zeta) \\
& \geq f(\xi)+\zeta \cdot D f(\xi) \\
& =f(D \widehat{a}(x))+D \varphi(x, t) \cdot D f(D \widehat{a}(x))
\end{aligned}
$$

for almost all $(x, t) \in \Omega_{T}$. Integrating this over $\Omega_{T}$ we get

$$
\begin{aligned}
\int_{\Omega_{T}} f(D v) \mathrm{d} z & =\int_{\Omega_{T}} f(D \widehat{a}+D \varphi) \mathrm{d} z \\
& \geq \int_{\Omega_{T}} f(D \widehat{a})+D \varphi \cdot D f(D \widehat{a}) \mathrm{d} z \\
& =\int_{\Omega_{T}} f(D \widehat{a})-\varphi \operatorname{div}_{x} D f(D \widehat{a}) \mathrm{d} z \\
& =\int_{\Omega_{T}} f(D \widehat{a})-(v-\widehat{a}) \operatorname{div}_{x} D f(D \widehat{a}) \mathrm{d} z,
\end{aligned}
$$

where the identity follows from the Gauss-Green theorem. By (6.4) and (6.5) it now suffices to show that

$$
\int_{\Omega_{T}}\left(g_{u}^{+}(x, \widehat{a})-\operatorname{div}_{x} D f(D \widehat{a})\right)(v-\widehat{a}) \mathrm{d} z \geq 0 .
$$

Recall that the condition $\left(\mathrm{H}_{h, \omega}\right)$ says that $c \geq d+\omega(|h|) \Longrightarrow g_{u}^{+}(y-h, c) \geq g_{u}^{+}(y, d)$ for all $h, y \in \mathbb{R}^{n}$ and $c, d \in \mathbb{R}$. 
We fix a point $z_{0} \in \Omega$ whose choice does not matter. Using the above with $c:=\widehat{a}(x)$, $d=\widehat{a}(x)-\omega(|h|), h:=z_{0}-x$ and $y:=z_{0}$, we obtain

$$
\begin{aligned}
g_{u}^{+}(x, \widehat{a}(x)) & \geq g_{u}^{+}\left(z_{0}, \widehat{a}(x)-\omega(|h|)\right) \\
& \geq g_{u}^{+}\left(z_{0}, a(x)-\omega(\operatorname{diam} \Omega)\right) \\
& \geq g_{u}^{+}\left(z_{0},-\|a\|_{L^{\infty}(\Omega)}-\omega(\operatorname{diam} \Omega)\right),
\end{aligned}
$$

where we also used that $\widehat{a} \geq a$ in $\Omega$. Since $v-\widehat{a} \geq 0$, condition (6.6) further reduces to showing that

$$
g_{u}^{+}\left(z_{0},-\|a\|_{L^{\infty}(\Omega)}-\omega(\operatorname{diam} \Omega)\right)-\operatorname{div}_{x} D f(D \widehat{a}(x)) \geq 0
$$

for almost all $x \in \Omega$. We observe that $D a$ is a constant vector and compute

$$
D \widehat{a}(x)=D\left(a(x)+\beta x_{1}\left(3 \operatorname{diam} \Omega-x_{1}\right)\right)=D a+3 \beta(\operatorname{diam} \Omega) e_{1}-2 \beta x_{1} e_{1}
$$

so that

$$
D^{2} \widehat{a}(x)=-2 \beta\left(e_{1} \otimes e_{1}\right) .
$$

Hence by chain rule and $\Phi$-uniform convexity of $f$ we have

$$
\begin{aligned}
-\operatorname{div}_{x} D f(D \widehat{a}(x)) & =-\operatorname{tr}\left(J_{D f \circ D \widehat{a}}(x)\right)=-\operatorname{tr}\left(J_{D f}(D \widehat{a}(x)) J_{D \widehat{a}}(x)\right) \\
& =2 \beta \operatorname{tr}\left(D^{2} f(D \widehat{a}(x)) e_{1} \otimes e_{1}\right) \\
& \geq 2 \beta \Phi(|D \widehat{a}(x)|) .
\end{aligned}
$$

Observe that

$$
\begin{aligned}
|D \widehat{a}(x)| & =\left|D a+3 \beta(\operatorname{diam} \Omega) e_{1}-2 \beta x_{1} e_{1}\right| \geq 3 \beta \operatorname{diam} \Omega-2 \beta x_{1}-|D a| \\
& =\beta\left(3 \operatorname{diam} \Omega-2 x_{1}\right)-|D a| .
\end{aligned}
$$

Thus we can make $|D \widehat{a}|$ arbitrarily large in $\Omega$ by taking large enough $\beta$. Using the condition $s \Phi(s) \rightarrow \infty$ as $s \rightarrow \infty$ (with $s=|D \widehat{a}|$ ), it follows that there exists $\beta=\beta\left(\Omega, \Phi, g,\|a\|_{W^{1, \infty}(\Omega)}\right)$ such that

$$
\begin{aligned}
2 \beta \Phi(|D \widehat{a}(x)|) & =\frac{2 \beta}{|D \widehat{a}(x)|}|D \widehat{a}(x)| \Phi(|D \widehat{a}(x)|) \\
& \geq \frac{2 \beta}{|D a|+5 \beta \operatorname{diam} \Omega}|D \widehat{a}(x)| \Phi(|D \widehat{a}(x)|) \\
& \geq\left|g_{u}^{+}\left(x_{0},-\|a\|_{L^{\infty}(\Omega)}-\omega(\operatorname{diam} \Omega)\right)\right| .
\end{aligned}
$$

The desired inequality (6.7) now follows from (6.8) and (6.9).

Proof of Corollary 2.9. By Corollary 2.5, it suffices to show that there exist Lipschitz continuous functions $l_{1}, l_{2} \in W_{u_{0}}^{1, p}(\Omega)$ such that

$$
l_{1} \leq u \leq l_{2} \text { in } \Omega_{T} .
$$


We will only construct the function $l_{2}$ as the construction of $l_{1}$ is analogical. Let $x_{0} \in \partial \Omega$. Since $\left.u_{0}\right|_{\partial \Omega}$ satisfies the bounded slope condition with a constant $Q$ and $u_{0}$ is Lipschitz in $\bar{\Omega}$, there is an affine function $a_{x_{0}}: \mathbb{R}^{n} \rightarrow \mathbb{R}$ such that

$$
u_{0}\left(x_{0}\right)=a_{x_{0}}\left(x_{0}\right) \text { and } u_{0} \leq a_{x_{0}} \text { in } \bar{\Omega},
$$

and additionally $\left|D a_{x_{0}}\right| \leq \max \left(Q,\left\|D u_{0}\right\|_{L^{\infty}(\Omega)}\right)$, see [4, Lemma 2.3]. Let $\widehat{a}_{x_{0}}$ be the variational super-solution given by Lemma 6.1. Then we have

$$
u_{0}\left(x_{0}\right)=a\left(x_{0}\right)=\widehat{a}_{x_{0}}\left(x_{0}\right) \text { and } u_{0} \leq a_{x_{0}} \leq \widehat{a}_{x_{0}} \text { in } \bar{\Omega} .
$$

Thus it follows from comparison principle (Lemma 3.3) that $u \leq \widehat{a}_{x_{0}}$ in $\bar{\Omega}_{T}$. Moreover, from the proof of Lemma 6.1 we see that $\left\|D \widehat{a}_{x_{0}}\right\|_{L^{\infty}(\Omega)}$ depends only on $\Phi, g, \Omega$, $\sup _{\partial \Omega}\left|u_{0}\right|$ and $\left|D a_{x_{0}}\right|$. In other words, the Lipschitz constant of $\widehat{a}_{x_{0}}$ is independent of $x_{0}$. Therefore the function

$$
l_{2}(x):=\inf _{x_{0} \in \partial \Omega} \widehat{a}_{x_{0}}(x), x \in \bar{\Omega},
$$

is a desired Lipschitz continuous upper bound for $u$. Note that $l_{2}$ is in fact Lipschitz since it is the infimum of a family of Lipschitz functions with a uniform Lipschitz constant.

\section{Declarations.}

\section{Funding}

R. Rainer has been supported by the FWF-Project P 31956 "Doubly Nonlinear Evolution Equations".

\section{Conflicts of interest/Competing interests}

Not applicable.

\section{Availability of data and material}

Not applicable.

\section{Code availability}

Not applicable. 


\section{Authors' contributions}

Each author contributed equally to the scientific value of this paper. All authors commented and took part in improvements on previous versions of the manuscript and approved the final version.

Funding Open access funding provided by Paris Lodron University of Salzburg.

Open Access This article is licensed under a Creative Commons Attribution 4.0 International License, which permits use, sharing, adaptation, distribution and reproduction in any medium or format, as long as you give appropriate credit to the original author(s) and the source, provide a link to the Creative Commons licence, and indicate if changes were made. The images or other third party material in this article are included in the article's Creative Commons licence, unless indicated otherwise in a credit line to the material. If material is not included in the article's Creative Commons licence and your intended use is not permitted by statutory regulation or exceeds the permitted use, you will need to obtain permission directly from the copyright holder. To view a copy of this licence, visit http://creativecommons.org/ licenses/by/4.0/.

\section{References}

[1] Bögelein, V., Dacorogna, B., Duzaar, F., Marcellini, P., Scheven, C.: Integral convexity and parabolic systems. SIAM J. Math. Anal. 52(2), 1489-1525 (2020)

[2] Bögelein, V., Duzaar, F., Marcellini, P.: Existence of evolutionary variational solutions via the calculus of variations. J. Differ. Equ. 256(12), 3912-3942 (2014)

[3] Bögelein, V., Duzaar, F., Marcellini, P., Scheven, C.: Doubly nonlinear equations of porous medium type. Arch. Ration. Mech. Anal. 229(2), 503-545 (2018)

[4] Bögelein, V., Duzaar, F., Marcellini, P., Signoriello, S.: Parabolic equations and the bounded slope condition. Ann. Inst. H. Poincaré Anal. Non Linéaire 34(2), 355-379 (2017)

[5] Bögelein, V., Stanin, T.: The one-sided bounded slope condition in evolution problems. Ann. Mat. Pura Appl. 4 199(2), 573-587 (2019)

[6] Bousquet, P.: Boundary continuity of solutions to a basic problem in the calculus of variations. Adv. Calc. Var. 3, 1-27 (2010)

[7] Bousquet, P.: Continuity of solutions of a problem in the calculus of variations. Calc. Var. 41, 413-433 (2011)

[8] Bousquet, P., Brasco, L.: Global Lipschitz continuity for minima of degenerate problems. Math. Ann. 366(3-4), 1403-1450 (2016)

[9] Bousquet, P., Clarke, F.: Local lipschitz continuity of solutions to a problem in the calculus of variations. J. Differ. Equ. 243, 489-503 (2007)

[10] Cellina, A.: On the bounded slope condition and the validity of the euler lagrange equation. SIAM J. Control Optim. 40, 1270-1279 (2001)

[11] Cellina, A.: Comparison results and estimates on the gradient without strict convexity. SIAM J. Control Optim. 46, 738-749 (2007)

[12] Clarke, F.: Continuity of solutions to a basic problem in the calculus of variations. Ann. Sc. Norm. Super. Pisa Cl. Sci. 4, 511-530 (2005) 
[13] Evans, L.C., Gariepy, R.F.: Measure Theory and Fine Properties of Functions. CRC Press, revised edition, (2015)

[14] Fiaschi, A., Treu, G.: The bounded slope condition for functionals depending on $x, u$, and $\nabla u$. SIAM J. Control Optim. 50(2), 991-1011 (2012)

[15] Giaquinta, M., Martinazzi, L.: An introduction to the regularity theory for elliptic systems, harmonic maps and minimal graphs, volume 2 of Appunti. Scuola Normale Superiore di Pisa (Nuova Serie) [Lecture Notes. Scuola Normale Superiore di Pisa (New Series)]. Edizioni della Normale, Pisa, (2005)

[16] Giusti, E.: Direct methods in the calculus of variations. World Scientific Publishing Co., Inc, River Edge, NJ (2003)

[17] Haar, A.: Über das Plateausche Problem. Math. Ann. 97(1), 124-158 (1927)

[18] Hardt, R., Zhou, X.: An evolution problem for linear growth functionals. Commun. Partial Differ. Equ. 19(11-12), 1879-1907 (1994)

[19] Hartman, P.: On the bounded slope condition. Pacific J. Math. 18, 495-511 (1966)

[20] Hartman, P., Nirenberg, L.: On spherical image maps whose jacobians do not change sign. Am. J. Math. 81, 901-920 (1959)

[21] Hartman, P., Stampacchia, G.: On some non-linear elliptic differential functional equation. Acta Math. 115, 271-310 (1966)

[22] Lichnewsky, A., Temam, R.: Pseudosolutions of the time-dependent minimal surface problem. J. Differ. Equ. 30(3), 340-364 (1978)

[23] Mariconda, C., Treu, G.: Lipschitz regularity for minima without strict convexity of the lagrangian. J. Differ. Equ. 243, 388-413 (2007)

[24] Mariconda, C., Treu, G.: Local lipschitz regularity of minima for a scalar problem of the calculus of variations. Commun. Contemp. Math. 10, 1129-1149 (2008)

[25] Mariconda, C., Treu, G.: Hölder regularity for a classical problem of the calculus of variations. Adv. Calc. Var. 2, 311-320 (2009)

[26] Mariconda, C., Treu, G.: A Haar-Rado type theorem for minimizers in Sobolev spaces. ESAIM Control Optim. Calc. Var., 17(4), (2011)

[27] Miranda, M.: Un teorema di esistenza e unicità per il problema dell'area minima in $n$ variabili. Ann. Scuola Norm. Sup. Pisa Cl. Sci. (3) 19, 233-249 (1965)

[28] Stampacchia, G.: On some regular multiple integral problems in the calculus of variations. Comm. Pure Appl. Math. 16, 383-421 (1963)

Publisher's Note Springer Nature remains neutral with regard to jurisdictional claims in published maps and institutional affiliations. 